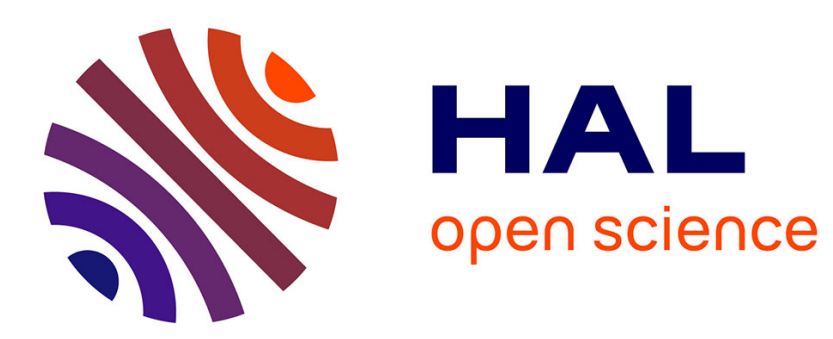

\title{
Pretransitional behaviour of a nematic liquid crystal in the isotropic phase
}

\author{
J. Fuchs, W. Burchard
}

\section{To cite this version:}

J. Fuchs, W. Burchard. Pretransitional behaviour of a nematic liquid crystal in the isotropic phase. Journal de Physique II, 1994, 4 (9), pp.1451-1456. 10.1051/jp2:1994102 jpa-00248053

\section{HAL Id: jpa-00248053 https://hal.science/jpa-00248053}

Submitted on 1 Jan 1994

HAL is a multi-disciplinary open access archive for the deposit and dissemination of scientific research documents, whether they are published or not. The documents may come from teaching and research institutions in France or abroad, or from public or private research centers.
L'archive ouverte pluridisciplinaire HAL, est destinée au dépôt et à la diffusion de documents scientifiques de niveau recherche, publiés ou non, émanant des établissements d'enseignement et de recherche français ou étrangers, des laboratoires publics ou privés. 
Classification

Physics Abstracts

$78.35-64.70 \mathrm{M}$

\title{
Short Communication
}

\section{Pretransitional behaviour of a nematic liquid crystal in the isotropic phase}

\author{
J. Fuchs and W. Burchard \\ Institute of Macromolecular Chemistry, University of Freiburg, Stefan-Meier-Str. 31, \\ 79104 Freiburg, Germany
}

(Received 23 March 1994, revised 14 June 1994, accepted 20 July 1994)

\begin{abstract}
Polarized and depolarized light scattering measurements have been performed from a nematic liquid crystalline material in the isotropic region, approaching the nematic phase transition. A critical static scattering intensity and a critical slowing-down of the orientational fluctuations were observed in a temperature range of about $10^{\circ} \mathrm{C}$ above the phase transition which followed a $\left(T-T_{\mathrm{c}}^{*}\right)^{-\gamma}$ dependence, but with an exponent slightly smaller than unity. The angular dependence of the polarized static scattering intensity differed in behaviour from other liquid crystals by the occurrence of a strong excess scattering at small scattering angles. These observations give evidence for spatial structural heterogeneities, spherical in shape and optically isotropic, in a bath of a nematic liquid crystalline system. This conclusion is confirmed by dynamic light scattering where in addition a slow mode appeared in the time correlation function which results from random migration of these heterogeneities.
\end{abstract}

\section{Introduction.}

At high temperatures a nematic liquid crystal forms a clear and isotropic liquid, but becomes highly turbid on cooling below a critical temperature. Such a phase transition mostly occurs with rodlike (in general optically anisotropic) molecules. In the isotropic phase the rods are randomly distributed; below the so-called clearing temperature the molecules assume a high order in orientation but not with respect to their center of mass. Molecules with their long axes in parallel alignment to each other cause an anisotropy of the dielectric constant. Therefore, an optical method like light scattering appears to be ideal for studying a nematic liquid phase transition.

The term "weakly first order" for the transition from the isotropic to the nematic state was introduced [1]. The effect of the "nearly second order" behaviour [2] is noticed by a strong increase of the scattered light and of the orientational fluctuations relaxation time when 
approaching the transition. The critical point is never achieved, because a first order phase transition takes place, typically $1 \mathrm{~K}$ above the critica. The behaviour in the isotropic state in the vicinity of the phase transition (pretransitional behaviour) can be well interpreted with a phenemenological model introduced by Landau and extended by de Gennes [3]. Only close to the phase transitional boundary, deviations from the theoretically predicted mean field behaviour were observed in various laboratories [4-6].

In this paper the pretransitional light scattering of a nematic liquid crystal with the following structure [7] was investigated:

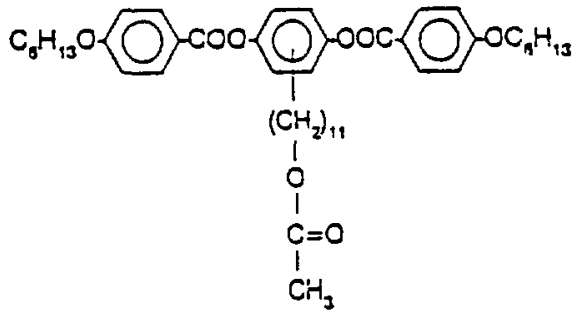

The far too high clearing temperature of the bare mesogene could be brought down to a reasonably low temperature $\left(45^{\circ} \mathrm{C}\right)$ by introducing a large aliphatic side chain to the hydrochinon unit and two short tails at the ends of the benzoate units. The polarized and the depolarized component of the scattered light and the time correlation function were recorded in dependence on temperature and scattering angle. The results are compared with the Landau-de Gennes theory in the with an exponent $\gamma$ that may deviate from unity.

The experimentally measured Rayleigh ratio is given by

$$
\begin{gathered}
R_{\Theta}^{\mathrm{VH}}=K \frac{k_{\mathrm{B}} T}{a\left(T-T_{\mathrm{c}}^{*}\right)^{\gamma}} \frac{1}{2}\left[\cos ^{2} \frac{\Theta}{2} \frac{1}{1+\xi_{\perp}^{2} q^{2}}+\sin ^{2} \frac{\Theta}{2} \frac{1}{1+\xi_{1}^{2} q^{2}}\right] \\
R_{\Theta}^{\mathrm{VV}}=K \frac{k_{\mathrm{B}} T}{a\left(T-T_{\mathrm{c}}^{*}\right)^{\gamma}} \frac{1}{2}\left[\frac{1}{3} \frac{1}{1+\xi_{\|}^{2} q^{2}}+\frac{1}{1+\xi_{1}^{2} q^{2}}\right]
\end{gathered}
$$

where $K=\frac{1}{(4 \pi \epsilon)^{2}} k_{f}^{4}\left(\Delta \epsilon_{\max }\right)^{2}$, and $\xi_{1}, \xi_{\perp}$ and $\xi_{\|}$are correlation lengths [2]. These are mostly small compared to the wavelength of the light, and are difficult to measure accurately [8]. The scattering geometry for the orientation of polarizer and analyzer is vertical-vertical (VV) for equation (1) and vertical-horizontal (VH) for equation (2).

\section{Experimental.}

The synthesis of the liquid crystalline material was performed similar to a description given in literature [7].

The resulting product was purified in a first step by flash chromatography [9] using dichloromethane as eluant. Thin layer chromatography with ether/ethyl acetate (9:1) revealed still impurities. A second flash chromatography was made with that solvent mixture. Finally the material was recrystallized in ether and a second time in ethanol.

The liquid crystalline phase behaviour and the transition temperatures were determined by differential scanning calorimetry and polarizing microscopy. The purified product had a melting temperature of $T_{\mathrm{m}}=52.0^{\circ} \mathrm{C}$ and displayed a nematic phase below $T_{\mathrm{c}}=45.0^{\circ} \mathrm{C}$.

The sample was made dust-free by filtration of its solution in benzene through a Millipore filter (MF-Millipore $0.45 \mu \mathrm{m}$ ) and freeze drying afterwards. Static and dynamic light scattering 

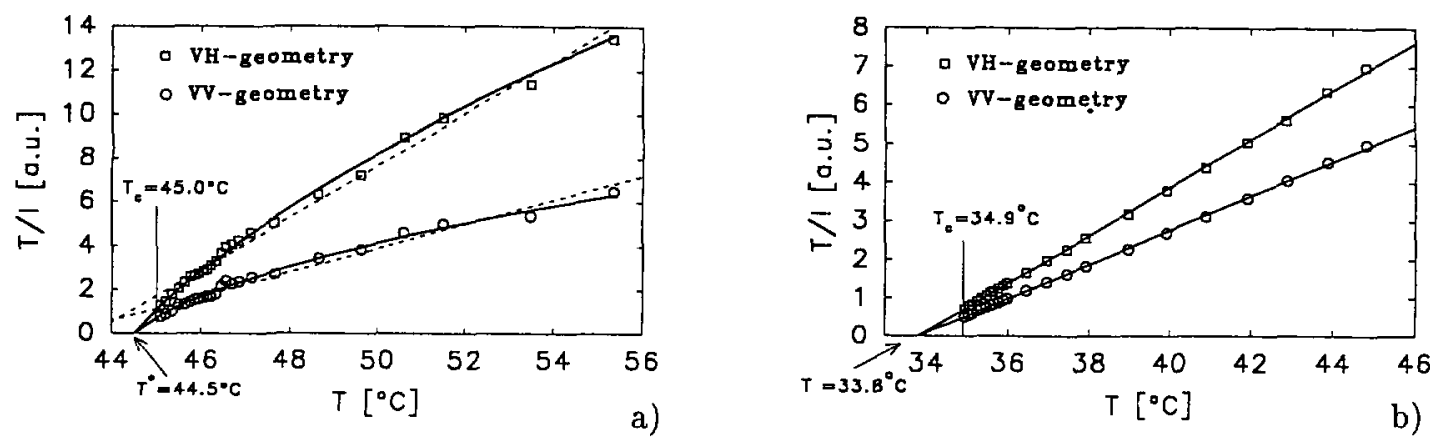

Fig. 1. - Temperature dependence of the inverse scattering intensity for the present (1a) and the 5CB (1b) sample. Linear behaviour was found by us for 5CB but significant curvature for the present sample. The full lines in figure $1 \mathrm{a}$ are according to equation (3) with $\gamma<1$.
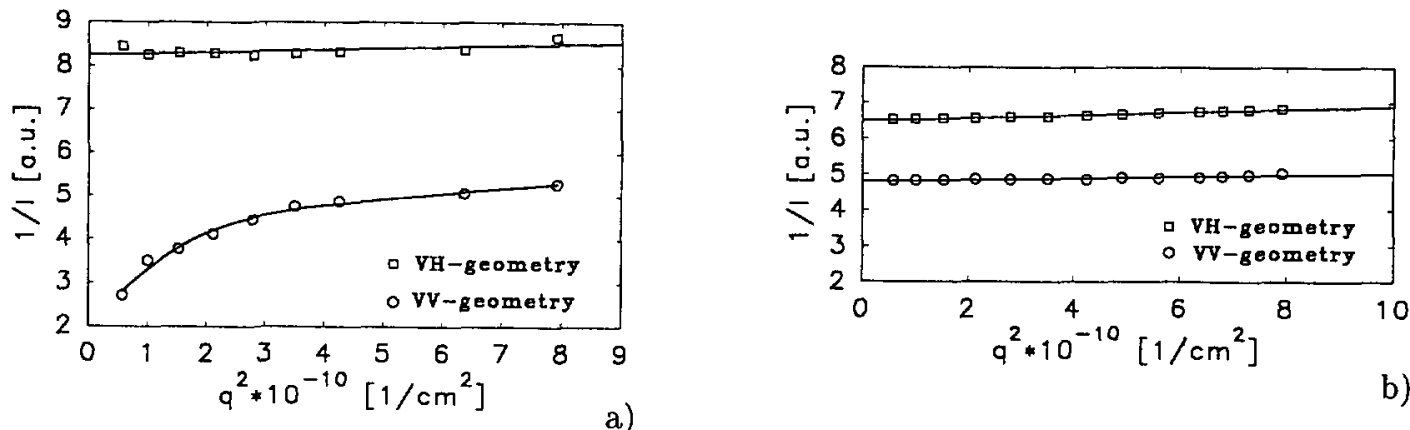

a)

b)

Fig. 2. - Note the curvature in the VV geometry for the present sample (2a) in contrast to the angular independence that was observed with 5 CB (2b).

measurements have been performed using an automatic ALV goniometer and an ALV Structurator/Correlator ALV 3000. The slow mode in the time correlation function was measured in the multiple- $\tau$ mode using 192 channels. A krypton ion laser was used as a light source with the wavelength $\lambda_{0}=647.1 \mathrm{~nm}$.

\section{Results and discussion.}

3.1 StATIC Light SCATTERING. - The intensity $I$ of the scattered light was detected at a constant angle $\Theta=90^{\circ}$ over a temperature range of $10 \mathrm{~K}$ above $T_{c}$. Figure 1 shows the temperature dependence of $T / I$ for the polarized and the depolarized scattering geometry. The full lines in figure 1a represent the best fit with equation (3) where the exponents are $\gamma_{\mathrm{VV}}=0.72$ and $\gamma_{\mathrm{VH}}=0.88$ for the $\mathrm{VV}$ and the $\mathrm{VH}$ geometry respectively.

$$
\frac{T}{I} \propto\left(T-T_{c}^{*}\right)^{\gamma}
$$

The dashed lines clearly show that linear regression fails which is in contrast to our measurment from 5CB (Fig. 1b).

Next, the angular dependence of the scattered light was checked. In figure $2 \mathrm{a}$ the reciprocal scattering intensity is plotted as a function of $q^{2}=\left(\frac{4 \pi}{\lambda} \sin \frac{\Theta}{2}\right)^{2}$. Similar to other liquid crystals, 
e.g. 5CB (Fig. 2b), no angular dependence was observed in the VH geometry but an unexpected rather strong low-angle scattering was obtained in the VV geometry.

This behaviour reminds of the scattering curves from semidilute polymer solutions, where the low-angle excess scattering was interpreted as being caused by cluster formation [10]. Therefore, the following equation was used for a fit of the curves resulting from a two component system

$$
I(q) \propto M(T) \exp \left(-\frac{R_{\mathrm{g}}^{2} q^{2}}{3}\right)+[1-M(T)] \frac{1}{\left(1+\xi^{2} q^{2}\right)}
$$

$M(T) \leq 1$ is an adjustable parameter, $R_{\mathrm{g}}$ the radius of gyration of the clusters and $\xi$ a correlation length. The first term represents a Guinier approximation for the large clusters. The second one corresponds to fluctuations according to a space correlation function $C(r)=$ $\frac{1}{r} \exp \left(-\frac{r}{\xi}\right)$, which describes the correlations in the approximation of the Landau-de Gennes theory $[1,3]$. In the whole temperature range from $46^{\circ} \mathrm{C}$ to $100^{\circ} \mathrm{C}$ no significant change in the radius of gyration $R_{\mathrm{g}} \approx 200 \mathrm{~nm}$ was found. Only in the range $1 \mathrm{~K}$ above the transition temperature $R_{\mathrm{g}}$ becomes smaller and reaches its lowest value with $R_{\mathrm{g}}=141 \mathrm{~nm}$. There was also almost no change in the magnitude of the correlation length $\xi \approx 16 \mathrm{~nm}$. According to the Landau approximation, the temperature dependence of $\xi$ should be of the form

$$
\xi=\xi_{0} \sqrt{\left(\frac{T_{c}^{*}}{T}-T_{c}^{*}\right)}
$$

where $\xi_{0}$ is a molecular length. This deviation from theory may result from the difficulty to determine accurately a correlation length of $16 \mathrm{~nm}$ with a wavelength of $647.1 \mathrm{~nm}$ of the light used. A second difficulty arises from the influence of the strong small angle excess scattering in the wide angle area. The problem is even more involved because of the temperature dependence of the amplitude $M(T)$. One realizes from figure 3 that $M(T)$ strongly decreases as the critical temperature is approached while the radius of gyration remained virtually unchanged. This is to say, the clusters become less visible since the orientational fluctuations start to dominate. In the high $T$ region $\left(T>55^{\circ} \mathrm{C}\right)$, however, $M$ seems to attain a constant value. Apparently the ratio of the cluster to the orientational fluctuations remains independent of the temperature. The prefactor $(1-M(T))$ represents the weighed influence of the orientational fluctuations

$$
I_{\text {Orient. }} \propto(1-M(T))
$$

In the pretransitional area a critical behaviour of $I_{\text {Orient. }}$ is expected

$$
\frac{1}{I_{\text {Orient. }}} \propto\left(T-T^{*}\right)
$$

as observed for other liquid crystalline samples $[5,6,11]$. Therefore, the critical point is achieved when the scattering contribution of the clusters become negligible $(M(T) \rightarrow 0)$. The plot of $[1 /(1-M(T))]$ against temperature $T$ gave a linear relationship, that could be extrapolated to unity (i.e. $M(T)=0$ ). The corresponding temperature would be then $T^{*}$. Linear regression (not shown) gave a critical temperature of $T^{*}=43.6^{\circ} \mathrm{C}$. This temperature is $0.9 \mathrm{~K}$ lower than determined from the plot in figure 1 where only the raw data at $\Theta=90^{\circ}$ were used, which to some extend contain contributions of the excess scattering. The latter extrapolation for $T^{*}$ has to be considered as being the more accurate, because the scattering intensities were freed from this excess scattering and correspond to the orientational fluctuations only. 


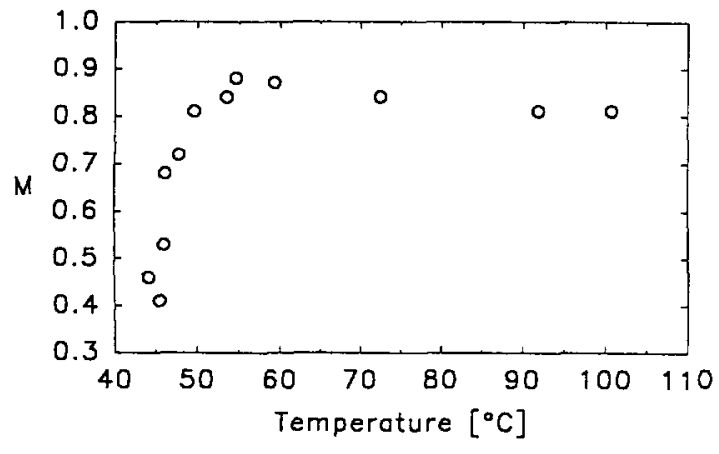

Fig. 3

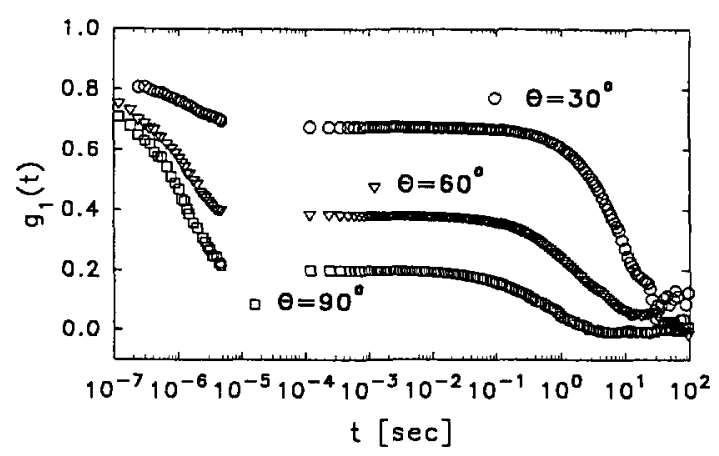

Fig. 4

Fig. 3. - The parameter $M$ (Eq. (4)) representing the scattering contribution caused by heterogeneities.

Fig. 4. - Polarized TCF $g_{1}(t)$ at three different scattering angles $\left(T=53.5^{\circ} \mathrm{C}\right)$.

3.2 Dynamic Light SCATtering. - Another indication for the presence of heterogeneities was obtained from dynamic light scattering. For the VV geometry figure 4 revealed a slow mode in addition to the expected fast relaxation process. The fast relaxation process was recorded in the single- $\tau$ the slow mode in the multiple- $\tau$ modes of the ALV-3000 correlator. The measurements is the reason for the gap when both time correlation functions (TCFs) are plotted in the same graph. The slow mode has typical diffusive character and could be fitted by a stretched exponential

$$
g_{1}(t)_{\text {slow }}=\exp \left[-\left(\frac{t}{\tau}\right)^{\beta}\right]
$$

with an exponent $\beta=0.85$ while the fast mode could be well described by a single exponential. The same relaxation process was found in the VH geometry, but no slow motion. This is in agreement with the angular independence of the static scattering curves. In figure $5(\Gamma=1 / \tau)$

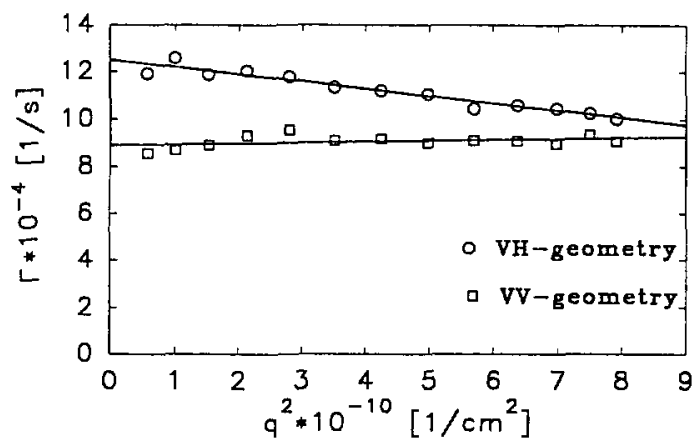

Fig. 5

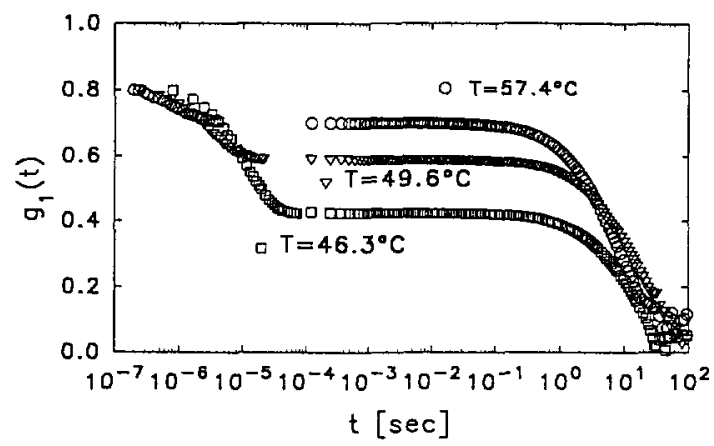

Fig. 6

Fig. 5. - Angular dependence of the reciprocal relaxation time for both scattering geometries at $T=46.1{ }^{\circ} \mathrm{C}$.

Fig. 6. - Polarized TCF recorded at three different temperatures and $\Theta=30^{\circ}$. 
is plotted versus $q^{2}$. Almost no angular dependence was found for the fast mode studied in the VV geometry; it is assigned to the collective orientational fluctuations of rods. The angular dependence of $\Gamma$ for the $\mathrm{VH}$ geometry is unexpected and unusual. The physical meaning of the negative slope of the straight line is not yet clear to us.

Information on the the temperature dependence of both relaxation processes is obtained from figure 6. The behaviour of the fast mode revealed a critical slowing down whereas the slow motion was only weakly affected by temperature.

\section{Conclusion.}

Low molecular weight liquid crystalline material studied so far exhibit mostly no or only a weak angular dependency of the scattered light in both the VH and VV geometry, even when the isotropic nematic phase transition is approached. In contrast to these thermotropic samples the system investigated in the present study exhibits a strong low angle excess scattering in the VV but not in the VH geometry. Simultaneously a slow mode is observed in the VV dynamic light scattering indicating the presence of heterogeneities. The excess scattering and the contribution of the slow motion become suppressed when the phase transition is approached. The effect is interpreted as being caused by dominating orientational fluctuations which became critically large at the phase boundary. In o caused by the long aliphatic chain attached to the mesogene. This long chain is certainly the main difference by that the present material deviates from the more common $n$ CB molecules. This idea will be followed in further studies of other thermotropic samples with varying length of aliphatic side chains.

\section{Acknowledgments.}

We thank Mrs. E. Stibal-Fischer for her kind help in preparing the material. The 5CB sample was kindly given to us by BDH Limited Poole, England. Financial support by the Deutsche Forschungsgemeinschaft (Sonderforschungsbereich 60) is gratefully acknowledged.

\section{References}

[1] de Gennes P.G., The Physics of Liquid Crystals, Chapter 2 (Clarendon Press, Oxford, 1974).

[2] Sheng P., Priestley E.B., Introduction to Liquid Crystals, Chapter 10 (Plenum Press, New York and London, 1971).

[3] de Gennes P.G., Mol. Cryst. Lıq. Cryst. 12 (1971) 193.

[4] Gramsbergen E.F., Longa L., de Jeu W.H., Phys. Rep. 135 (1986) 195.

[5] Stinson T.W., Litster J.D., Phys. Rev. Lett. 25 (1970) 503.

[6] Zink H., de Jeu W.H., Mol. Cryst. Liq. Cryst. 124 (1985) 287.

[7] Hessel F., Herr R.P., Finkelmann H., Makromol. Chem. 188 (1987) 1597.

[8] Stinson T.W., Litster J.D., Phys. Rev. Lett. 30 (1973) 688.

[9] Still W.C., Kahn M., Mitra A., J. Org. Chem. 43 (1978) 2923.

[10] Burchard W., Applied Fibre Science, Vol. 1, Chapter 10 (Academic Press, London, 1978).

[11] Stinson T.W., Litster J.D., Clark N.A., J. Phys. Colloq. France 33 (1972) C1-69. 